\title{
REGULARITY OF SOME NONLINEAR QUANTITIES ON SUPERHARMONIC FUNCTIONS IN LOCAL HERZ-TYPE HARDY SPACES
}

\author{
Dashan Fan, Shanzhen Lu* and Dachun Yang*
}

\begin{abstract}
In this paper, the authors introduce a kind of local Hardy spaces in $\mathbb{R}^{n}$ associated with the local Herz spaces. Then the authors investigate the regularity in these local Hardy spaces of some nonlinear quantities on superharmonic functions on $\mathbb{R}^{2}$. The main results of the authors extend the corresponding results of Evans and Müller in a recent paper.
\end{abstract}

\section{Introduction}

In recent years, Hardy space methods have lead to remarkable progress on nonlinear partial differential equations with critical growth; see [3], $[\mathbf{1 5}],[\mathbf{1}]$ and [16]. The main reason is as follows: a typical difficulty when studying such equations is that the nonlinear term is a priori only known to be in $L^{1}\left(\mathbb{R}^{n}\right)$ in which there is no good elliptic theory; however, there is a well-established regularity theory in a particular subspace of $L^{1}\left(\mathbb{R}^{n}\right)$, namely the standard Hardy space $\mathcal{H}^{1}\left(\mathbb{R}^{n}\right)$. In other words, Calderón-Zygmund singular integral operators are not bounded on $L^{1}\left(\mathbb{R}^{n}\right)$; but, they are indeed bounded on $\mathcal{H}^{1}\left(\mathbb{R}^{n}\right)$ if they satisfy the vanishing moment condition; see [5].

Keywords. Hardy spaces, Herz space, superharmonic function.

1991 Mathematics subject classifications: Primary 42B30; Secondary 35B65.

${ }^{*}$ The second and the third authors were supported in part by the NNSF and the SECF of China. 
Moreover, Coifman, Meyer, Lions and Semmes in [1] proved that certain nonlinear quantities mentioned above are in fact in $\mathcal{H}^{1}\left(\mathbb{R}^{n}\right)$. In particular, L. C. Evans and S. Müller in [3] established the local boundedness in standard Hardy space $\mathcal{H}^{1}\left(\mathbb{R}^{n}\right)$ of some nonlinear quantities on the weakly superharmonic functions. Applying their results to the two-dimensional Euler equations with nonnegative vorticity, Evans and Müller gave a new proof of Delort's results in [2].

On the other hand, a theory of the Hardy spaces associated with Herz spaces has been developed considerably in recent years; see [6], [11] and [12]. These Herz-type Hardy spaces are good substitutes of the usual Hardy spaces when studying boundedness of non-translation invariant operators (see [13]) and can be regarded as the local version at the origin of the usual Hardy spaces. There is also a good regularity theory in these spaces; see [12] and [14]. However, it is still not clear how to apply the theory on these spaces to partial differential equations. The main purpose of this paper is to establish a local version at the origin of Evans and Müller result in [3]. That is, we will study the local boundedness on the Hardy spaces associated with Herz spaces of some nonlinear quantities on weakly superharmonic functions. Our main results also extend the corresponding results of Evans and Müller, Theorem 1.1 and Theorem 5.4 in [3]. We hope this will be helpful to finding more applications of the Herz-type Hardy spaces in the study on partial differential equations; see also [14].

Let us first introduce some definitions.

For $k \in \mathbb{Z}$, let $B_{k}=B\left(0,2^{k}\right)=\left\{x \in \mathbb{R}^{n}:|x| \leq 2^{k}\right\}$ and $A_{k}=B_{k} \backslash B_{k-1}$. We denote the characteristic function of $A_{k}$ by $\chi_{k}$.

Definition 1 ([8]). Let $\alpha \in \mathbb{R}$ and $0<p, q<\infty . f \in L_{\text {loc }}^{q}\left(\mathbb{R}^{n} \backslash\{0\}\right)$ is said to belong to Herz space $\dot{K}_{q}^{\alpha, p}\left(\mathbb{R}^{n}\right)$, if

$$
\|f\|_{\dot{K}_{q}^{\alpha, p}\left(\mathbb{R}^{n)}\right.} \equiv\left\{\sum_{k=-\infty}^{\infty} 2^{k \alpha p}\left\|f \chi_{k}\right\|_{L^{q}\left(\mathbb{R}^{n}\right)}^{p}\right\}^{1 / p}<\infty .
$$

We introduce the local Herz spaces as follows.

Definition 2. Let $\alpha \in \mathbb{R}$ and $0<p, q<\infty . f \in L_{\mathrm{loc}}^{q}\left(\mathbb{R}^{n} \backslash\{0\}\right)$ is said to belong to local Herz space $\dot{K}_{q, \text { loc }}^{\alpha, p}\left(\mathbb{R}^{n} \backslash\{0\}\right)$ if for every $\phi \in C_{c}^{\infty}\left(\mathbb{R}^{n}\right)$, $\phi f \in \dot{K}_{q}^{\alpha, p}\left(\mathbb{R}^{n}\right)$. 
Obviously, by the above definition, $f \in \dot{K}_{q, \text { loc }}^{\alpha, p}\left(\mathbb{R}^{n}\right)$ is equivalent to the fact that for any $k \in \mathbb{Z}, \chi_{B_{k}} f \in \dot{K}_{q}^{\alpha, p}\left(\mathbb{R}^{n}\right)$. It is also easy to observe that $f \in \dot{K}_{q, \text { loc }}^{\alpha, p}\left(\mathbb{R}^{n}\right)$ if and only if for any $k \in \mathbb{N}, \chi_{B_{k}} f \in \dot{K}_{q}^{\alpha, p}\left(\mathbb{R}^{n}\right)$. We easily verify that $\dot{K}_{q, \text { loc }}^{n(1 / p-1 / q), p}\left(\mathbb{R}^{n}\right) \varsubsetneqq L_{\text {loc }}^{p}\left(\mathbb{R}^{n}\right)$ when $q>p \geq 1$ and $\dot{K}_{p, \text { loc }}^{0, p}\left(\mathbb{R}^{n}\right)=L_{\text {loc }}^{p}\left(\mathbb{R}^{n}\right)$.

Now choose $\eta: \mathbb{R}^{n} \longrightarrow \mathbb{R}$ to be any smooth function satisfying

$$
\operatorname{supp} \eta \subseteq B(0,1) \quad \text { and } \quad \int_{B(0,1)} \eta(x) d x=1 .
$$

If $f \in L^{1}\left(\mathbb{R}^{n}\right)$, we write

$$
f^{*}(x) \equiv \sup _{r \in(0, \infty)}\left|\frac{1}{r^{n}} \int_{\mathbb{R}^{n}} f(y) \eta\left(\frac{x-y}{r}\right) d y\right|
$$

In [11] (and independently in [6]), S. Lu and D. Yang introduce the following Herz-type Hardy spaces, $\mathcal{H} \dot{K}_{q}^{n(1-1 / q), 1}\left(\mathbb{R}^{n}\right)$, which can be regarded as the local version at the origin of the standard Hardy space, $\mathcal{H}^{1}\left(\mathbb{R}^{n}\right)$, studied by C. Fefferman and E. M. Stein in [5].

Definition 3 ([11]). Let $1 \leq q<\infty . f \in L^{1}\left(\mathbb{R}^{n}\right)$ is said to belong to the space $\mathcal{H} \dot{K}_{q}^{n(1-1 / q), 1}\left(\mathbb{R}^{n}\right)$ if $f^{*} \in \dot{K}_{q}^{n(1-1 / q), 1}\left(\mathbb{R}^{n}\right)$. Moreover, we define

$$
\|f\|_{\mathcal{H} \dot{K}_{q}^{n(1-1 / q), 1}\left(\mathbb{R}^{n}\right)} \equiv\left\|f^{*}\right\|_{\dot{K}_{q}^{n(1-1 / q), 1}\left(\mathbb{R}^{n}\right)} .
$$

According to Lu-Yang [12], this definition does not depend on the particular choice of $\eta$. Obviously, when $q=1, \mathcal{H} \dot{K}_{q}^{n(1-1 / q), 1}\left(\mathbb{R}^{n}\right)=$ $\mathcal{H}^{1}\left(\mathbb{R}^{n}\right)$, the standard Hardy space studied by Fefferman and Stein in $[5]$.

In [4], Fan and Yang introduce the local version, the space $h \dot{K}_{q}^{n(1-1 / q), 1}\left(\mathbb{R}^{n}\right)$, of the space $\mathcal{H} \dot{K}_{q}^{n(1-1 / q), 1}\left(\mathbb{R}^{n}\right)$ in Goldberg's sense [7]. For $f \in L_{\text {loc }}^{1}\left(\mathbb{R}^{n}\right)$, we will hereafter set

$$
f^{* *}(x)=\sup _{0<r \leq 1}\left|\frac{1}{r^{n}} \int_{\mathbb{R}^{n}} f(y) \eta\left(\frac{x-y}{r}\right) d y\right| .
$$


Definition 4 ([4]). Let $1 \leq q<\infty$. The space $h \dot{K}_{q}^{n(1-1 / q), 1}\left(\mathbb{R}^{n}\right)$ is defined by

$$
h \dot{K}_{q}^{n(1-1 / q), 1}\left(\mathbb{R}^{n}\right) \equiv\left\{f \in L^{1}\left(\mathbb{R}^{n}\right): f^{* *} \in \dot{K}_{q}^{n(1-1 / q), 1}\left(\mathbb{R}^{n}\right)\right\} .
$$

Moreover, in this case, we set

$$
\|f\|_{h \dot{K}_{q}^{n(1-1 / q), 1}\left(\mathbb{R}^{n}\right)} \equiv\left\|f^{* *}\right\|_{\dot{K}_{q}^{n(1-1 / q), 1}\left(\mathbb{R}^{n}\right)}
$$

It is clearly that when $q=1, h \dot{K}_{q}^{n(1-1 / q), 1}\left(\mathbb{R}^{n}\right)=h^{1}\left(\mathbb{R}^{n}\right)$, the local Hardy space introduced by Goldberg in [7]. By the results in [4], we also know that the above definition does not depend on the special choice of $\eta$. Now, we introduce another local version $\mathcal{H}_{\text {loc }} K_{q}^{n(1-1 / q), 1}\left(\mathbb{R}^{n}\right)$ of the space $\mathcal{H} \dot{K}_{q}^{n(1-1 / q), 1}\left(\mathbb{R}^{n}\right)$ as follows.

Definition 5. Let $1 \leq q<\infty . f \in L_{\text {loc }}^{1}\left(\mathbb{R}^{n}\right)$ is said to belong to the space $\mathcal{H}_{\mathrm{loc}} \dot{K}_{q}^{n(1-1 / q), 1}\left(\mathbb{R}^{n}\right)$ if for any $\phi \in C_{c}^{\infty}\left(\mathbb{R}^{n}\right), \phi f \in h \dot{K}_{q}^{n(1-1 / q), 1}\left(\mathbb{R}^{n}\right)$.

In the next section, we will prove the following equivalent definition of the space $\mathcal{H}_{\text {loc }} \dot{K}_{q}^{n(1-1 / q), 1}\left(\mathbb{R}^{n}\right)$.

Proposition 1. Let $1 \leq q<\infty$. Then $f \in \mathcal{H}_{\mathrm{loc}} \dot{K}_{q}^{n(1-1 / q), 1}\left(\mathbb{R}^{n}\right)$ if and only if for any $k \in \mathbb{Z}, \chi_{B_{k}} f^{* *} \in \dot{K}_{q}^{n(1-1 / q), 1}\left(\mathbb{R}^{n}\right)$, which is also equivalent to the fact that $f^{* *} \in \dot{K}_{q, \text { loc }}^{n(1-1 / q), 1}\left(\mathbb{R}^{n}\right)$.

We remark that when $q=1, \mathcal{H}_{\text {loc }} \dot{K}_{q}^{n(1-1 / q), 1}\left(\mathbb{R}^{n}\right)=\mathcal{H}_{\text {loc }}^{1}\left(\mathbb{R}^{n}\right)$, the local Hardy space introduced by Evans and Müller in [3].

To state our main theorem, we still need to introduce the local Herztype Sobolev space $H_{\mathrm{loc}}^{1} \dot{K}_{q}^{n(1 / 2-1 / q), 2}\left(\mathbb{R}^{n}\right)$; see also [14].

Definition 6. Let $1 \leq q<\infty$. We call $u \in H_{\mathrm{loc}}^{1} \dot{K}_{q}^{n(1 / 2-1 / q), 2}\left(\mathbb{R}^{n}\right)$ if $u$ and its distributional first partial derivatives $u_{x_{1}}, \ldots, u_{x_{n}}$ belong to the space $\dot{K}_{q, \text { loc }}^{n(1 / 2-1 / q), 2}\left(\mathbb{R}^{n}\right)$.

Obviously, when $q=2, H_{\mathrm{loc}}^{1} \dot{K}_{q}^{n(1 / 2-1 / q), 2}\left(\mathbb{R}^{n}\right)=H_{\mathrm{loc}}^{1}\left(\mathbb{R}^{n}\right)$, the standard local Sobolev space.

Now, it is a position to state our main theorem. 
Theorem 1. Let $1<q<\infty, u \in H_{\mathrm{loc}}^{1} \dot{K}_{2 q}^{1-1 / q, 2}\left(\mathbb{R}^{2}\right)$ be a weak solution of the partial differential equation

$$
-\triangle u=w \quad \text { in } \quad \mathbb{R}^{2} \text {. }
$$

where $w \in \dot{K}_{q, \text { loc }}^{2(1-1 / q), 1}\left(\mathbb{R}^{2}\right)$ and

$$
w \geq 0
$$

Then

$$
u_{x_{1}} u_{x_{2}}, u_{x_{1}}^{2}-u_{x_{2}}^{2} \in \mathcal{H}_{\mathrm{loc}} \dot{K}_{q}^{2(1-1 / q), 1}\left(\mathbb{R}^{2}\right) .
$$

In addition, for each $\phi \in C_{c}^{\infty}\left(\mathbb{R}^{2}\right)$, we have the estimate

$$
\begin{aligned}
\left\|\phi u_{x_{1}} u_{x_{2}}\right\|_{h \dot{K}_{q}^{2(1-1 / q), 1}\left(\mathbb{R}^{2}\right)}+\left\|\phi\left(u_{x_{1}}^{2} u_{x_{2}}^{2}\right)\right\|_{h \dot{K}_{q}^{2(1-1 / q), 1}\left(\mathbb{R}^{2}\right)} \\
\leq c\left\|\chi_{B(0, R)} \mid D u\right\|_{\dot{K}_{2 q}^{1-1 / q, 2}\left(\mathbb{R}^{2}\right)}
\end{aligned}
$$

for some constant $c$ and some radius $R$ depending only on $\phi$.

If we take $q=1$, then Theorem 1 is just Theorem 1.1 in [3]. Thus, Theorem 1 is a generalization of Theorem 1.1 in $[\mathbf{3}]$. In fact, Theorem 1 can be regarded as some kind local version of that theorem at the origin. See also Semmes [16] for several different versions of Evans and Müller's theorem.

It has been pointed by Evans and Müller in [3] that without the sign condition (1.1), Theorem 1 is false. However, in the radical case, the nonnegativity of $w$ is not required. To be precise, we have

Theorem 2. Let $1<q, q_{1}, q_{2}<\infty, u \in H_{\mathrm{loc}}^{1} \dot{K}_{q}^{2\left(1 / 2-1 / q_{1}\right), 2}\left(\mathbb{R}^{2}\right)$ be $a$ weak solution of the partial differential equation

$$
-\triangle u=w \quad \text { in } \quad \mathbb{R}^{2}
$$

with $w \in \dot{K}_{q_{2}, \text { loc }}^{2\left(1-1 / q_{2}\right), 1}\left(\mathbb{R}^{2}\right)$ and $u(x)=u(r), w(x)=w(r)$ for $r=|x|$. Then $u_{x_{1}} u_{x_{2}}, u_{x_{1}}^{2}-u_{x_{2}}^{2} \in \mathcal{H}_{\mathrm{loc}} \dot{K}_{q}^{2(1-1 / q), 1}\left(\mathbb{R}^{2}\right)$. Furthermore, for each $\phi \in C_{c}^{\infty}\left(\mathbb{R}^{2}\right)$, we have the estimate

$$
\begin{aligned}
& \left\|\phi u_{x_{1}} u_{x_{2}}\right\|_{h \dot{K}_{q}^{2(1-1 / q), 1}\left(\mathbb{R}^{2}\right)}+\left\|\phi\left(u_{x_{1}}^{2}-u_{x_{2}}^{2}\right)\right\|_{h \dot{K}_{q}^{2(1-1 / q), 1}\left(\mathbb{R}^{2}\right)} \\
& \leq c\left(\left\|\chi_{B_{k}} \mid D u\right\|_{\dot{K}_{q_{1}}^{2\left(1 / 2-1 / q_{1}\right), 2}\left(\mathbb{R}^{2}\right)}^{2}+\left\|\chi_{B_{k}} w\right\|_{\dot{K}_{q_{2}}^{2\left(1-1 / q_{2}\right), 1}\left(\mathbb{R}^{2}\right)}^{2}\right),
\end{aligned}
$$

where $k \in \mathbb{N}$ depends only on $\phi$. 
If we choose $q=1, q_{1}=2$ and $q_{2}=1$, we recover Theorem 5.4 in [3].

Finally, we point that we also obtain a local version on Herz-type Hardy spaces $h \dot{K}_{q}^{n(1-1 / q), 1}\left(\mathbb{R}^{n}\right)$ of Jones-Journé's result on convergence a.e. and convergence in the distribution sense for a sequence bounded in the Hardy space $\mathcal{H}^{1}\left(\mathbb{R}^{n}\right)$; see $[\mathbf{9}],[\mathbf{1}]$ and see also [3] for the local version on $h^{1}\left(\mathbb{R}^{n}\right)$.

Proposition 2. Let $1<q<\infty$. Suppose $\left\{f_{k}\right\}_{k=1}^{\infty}$ is bounded in $h \dot{K}_{q}^{n(1-1 / q), 1}\left(\mathbb{R}^{n}\right)$ and $f_{k} \rightarrow f$, a.e., as $k \rightarrow \infty$, for $f \in L_{\mathrm{loc}}^{1}\left(\mathbb{R}^{n}\right)$. Then $f \in h \dot{K}_{q}^{n(1-1 / q), 1}\left(\mathbb{R}^{n}\right)$ and

$$
f_{k} \rightarrow f
$$

in the sense of distributions.

If we take $q=1$, Proposition 2 is just Theorem 3.1 in [3].

\section{Proofs of Theorems}

We begin with the proof of Proposition 1.

Proof of Proposition 1: When $q=1$, this proposition is just Lemma 5.1 in $[3]$. Now we restrict that $1<q<\infty$. Suppose $f \in \mathcal{H}_{\text {loc }} \dot{K}_{q}^{n(1-1 / q), 1}\left(\mathbb{R}^{n}\right)$. For any $k \in \mathbb{Z}$, we choose $\phi \in C_{c}^{\infty}\left(\mathbb{R}^{n}\right)$ such that $\phi(x)=1$ when $x \in$ $B\left(0,2^{k}+1\right)$. Then, if $x \in B_{k}$,

$$
\begin{aligned}
(\phi f)^{* *}(x) & =\sup _{0<r \leq 1}\left|\frac{1}{r^{n}} \int_{\mathbb{R}^{n}} \eta\left(\frac{x-y}{r}\right) \phi(y) f(y) d y\right| \\
& =\sup _{0<r \leq 1}\left|\frac{1}{r^{n}} \int_{\mathbb{R}^{n}} \eta\left(\frac{x-y}{r}\right) f(y) d y\right| \\
& =f^{* *}(x) .
\end{aligned}
$$

Thus,

$$
\begin{aligned}
\left\|\chi_{B_{k}} f^{* *}\right\|_{\dot{K}_{q}^{n(1-1 / q), 1}\left(\mathbb{R}^{n}\right)} & =\left\|\chi_{B_{k}}(\phi f)^{* *}\right\|_{\dot{K}_{q}^{n(1-1 / q), 1}\left(\mathbb{R}^{n}\right)} \\
& \leq\left\|(\phi f)^{* *}\right\|_{\dot{K}_{q}^{n(1-1 / q), 1}\left(\mathbb{R}^{n}\right)} \\
& <\infty .
\end{aligned}
$$

That is, $\chi_{B_{k}} f^{* *} \in \dot{K}_{q}^{n(1-1 / q), 1}\left(\mathbb{R}^{n}\right)$. 
Now suppose $\chi_{B_{k}} f^{* *} \in \dot{K}_{q}^{n(1-1 / q), 1}\left(\mathbb{R}^{n}\right)$ for each $k \in \mathbb{Z}$. Fix any $\phi \in C_{c}^{\infty}\left(\mathbb{R}^{n}\right)$ with supp $\phi \subseteq B(0, R)$. Choose $l_{0} \in \mathbb{N}$ such that $2^{l_{0}-1} \leq$ $R+1<2^{l_{0}}$. Observe first that

$$
(\phi f)^{* *}(x)=\sup _{0<r \leq 1}\left|\frac{1}{r^{n}} \int_{\mathbb{R}^{n}} \eta\left(\frac{x-y}{r}\right) \phi(y) f(y) d y\right|
$$

vanishes if $x \in \mathbb{R}^{n} \backslash B(0, R+1)$. If $x \in B(0, R+1)$, by (5.2) in [3, p. 215], we have

$$
\begin{aligned}
(\phi f)^{* *}(x) & \leq c\left(f^{* *}(x)+\sup _{0<r \leq 1} \frac{1}{r^{n-1}} \int_{B(x, r)}|f(y)| d y\right) \\
& \leq c\left(f^{* *}(x)+\sum_{l=-\infty}^{l_{0}+1} \int_{\mathbb{R}^{n}} f_{l}(x-y)|y|^{1-n} \chi_{B_{0}}(y) d y\right),
\end{aligned}
$$

where $f_{l}(x)=|f(x)| \chi_{l}(x)$. Let $\bar{g}_{l}(x) \equiv \int_{\mathbb{R}^{n}} f_{l}(x-y)|y|^{1-n} \chi_{B_{0}}(y) d y$ and

$$
g(x)=\sum_{l=-\infty}^{l_{0}+1} \bar{g}_{l}(x) .
$$

Then,

$$
\begin{aligned}
\left\|(\phi f)^{* *}\right\|_{\dot{K}_{q}^{n(1-1 / q), 1}\left(\mathbb{R}^{n}\right)} \leq c\left\|\chi_{B_{l_{0}}} f^{* *}\right\|_{\dot{K}_{q}^{n(1-1 / q), 1}\left(\mathbb{R}^{n}\right)} & \\
& +c\left\|\chi_{B_{l_{0}}} g\right\|_{\dot{K}_{q}^{n(1-1 / q), 1}\left(\mathbb{R}^{n}\right)} .
\end{aligned}
$$

By the hypothesis, we know that $\left\|\chi_{B_{l_{0}}} f^{* *}\right\|_{\dot{K}_{q}^{n(1-1 / q), 1}\left(\mathbb{R}^{n}\right)}<\infty$. We still need to show that $\left\|\chi_{B_{l_{0}}} g\right\|_{\dot{K}_{q}^{n(1-1 / q), 1}\left(\mathbb{R}^{n}\right)}<\infty$. In fact, we have

$$
\begin{aligned}
\left\|\chi_{B_{l_{0}}} g\right\|_{\dot{K}_{q}^{n(1-1 / q), 1}\left(\mathbb{R}^{n)}\right)} & \leq \sum_{l=-\infty}^{l_{0}+1} \sum_{k=-\infty}^{l_{0}} 2^{k n(1-1 / q)}\left\|\bar{g}_{l} \chi_{k}\right\|_{L^{q}\left(\mathbb{R}^{n}\right)} \\
& =\sum_{l=-\infty}^{l_{0}+1} \sum_{k=-\infty}^{\min \left\{l+2, l_{0}\right\}} \cdots+\sum_{l=-\infty}^{l_{0}+1} \sum_{k=l+3}^{l_{0}} \cdots \\
& \equiv I_{1}+I_{2} .
\end{aligned}
$$


For $I_{1}$, by the trivial estimate $\left\|\bar{g}_{l} \chi_{k}\right\|_{L^{q}\left(\mathbb{R}^{n}\right)} \leq c\left\|f_{l}\right\|_{L^{q}\left(\mathbb{R}^{n}\right)}$ and the hypothesis, we obtain

$$
\begin{aligned}
I_{1} & \leq c \sum_{l=-\infty}^{l_{0}+1}\left\|f_{l}\right\|_{L^{q}\left(\mathbb{R}^{n}\right)}\left(\sum_{k=-\infty}^{l+2} 2^{k n(1-1 / q)}\right) \\
& \leq c \sum_{l=-\infty}^{l_{0}+1} 2^{l n(1-1 / q)}\left\|f_{l}\right\|_{L^{q}\left(\mathbb{R}^{n}\right)} \\
& \leq c\left\|\chi_{B_{l_{0}+1}} f^{* *}\right\|_{\dot{K}_{q}^{n(1-1 / q), 1}\left(\mathbb{R}^{n}\right)} \\
& <\infty .
\end{aligned}
$$

To estimate $I_{2}$, we first note that if $l \geq 0$ and $k \geq l+2$, then $\bar{g}_{l}(x) \equiv 0$ when $x \in A_{k}$. Thus,

$$
I_{2}=\sum_{l=-\infty}^{-1} \sum_{k=l+3}^{2} 2^{k n(1-1 / q)}\left\|\bar{g}_{l} \chi_{k}\right\|_{L^{q}\left(\mathbb{R}^{n}\right)} .
$$

Now, since $l \leq-1$ and $k \geq l+3$, for $x \in A_{k}$, we have

$$
\begin{aligned}
\bar{g}_{l}(x) & =\int_{\mathbb{R}^{n}}\left|f_{l}(x-y)\right||y|^{1-n} \chi_{B_{0}}(y) d y \\
& \leq \int_{3|x| \leq 4|y| \leq 5|x|}\left|f_{l}(x-y)\right||y|^{1-n} \chi_{B_{0}}(y) d y \\
& \leq c 2^{k(1-n)}\left\|f_{l}\right\|_{L^{1}\left(\mathbb{R}^{n}\right)} .
\end{aligned}
$$

By substituting this into $I_{2}$, we obtain

$$
\begin{aligned}
I_{2} & \leq c \sum_{l=-\infty}^{-1} \sum_{k=l+3}^{2} 2^{k}\left\|f_{l}\right\|_{L^{1}\left(\mathbb{R}^{n}\right)} \\
& \leq c \sum_{l=-\infty}^{-1} 2^{l n(1-1 / q)}\left\|f \chi_{l}\right\|_{L^{q}\left(\mathbb{R}^{n}\right)} \\
& \leq c\left\|\chi_{B_{-1}} f^{* *}\right\|_{\dot{K}_{q}^{n(1-1 / q), 1}\left(\mathbb{R}^{n}\right)} \\
& <\infty .
\end{aligned}
$$

This finishes the proof of Proposition 1. 
Proof of Theorem 1: Fix $R>8$. Let $B(0, R)$ denote the closed ball with center 0 and radius $R$, and set

$$
v(x)=-\frac{1}{2 \pi} \int_{B(0, R)} w(y) \log (|x-y|) d y .
$$

Then

$$
\theta=u-v \quad \text { is harmonic within } B(0, R)
$$

and

$$
v_{x_{i}}(x)=\frac{1}{2 \pi} \int_{B(0, R)} w(y) \frac{x_{i}-y_{i}}{|x-y|^{2}} d y, \quad i=1,2 .
$$

Choosing $\eta \in C_{c}^{\infty}\left(\mathbb{R}^{2}\right)$ satisfying

$$
\operatorname{supp} \eta \subseteq B(0,1), \quad \int_{B(0,1)} \eta(x) d x=1, \quad \eta \geq 0 .
$$

Let us also fix any point $x_{0} \in \mathbb{R}^{2}$. Consider then for $0<r \leq 1$, the expression

$$
\begin{aligned}
A= & \frac{1}{r^{2}} \int_{\mathbb{R}^{2}} v_{x_{1}}(x) v_{x_{2}}(x) \eta\left(\frac{x-x_{0}}{r}\right) d x \\
= & \frac{1}{4 \pi^{2} r^{2}} \int_{B(0, R)} \int_{B(0, R)} w(y) w(z) \\
& \left(\int_{\mathbb{R}^{2}} \frac{x_{1}-y_{1}}{|x-y|^{2}} \frac{x_{2}-z_{2}}{|x-z|^{2}} \eta\left(\frac{x-x_{0}}{r}\right) d x\right) d y d z
\end{aligned}
$$

by (2.2). We change variables by replacing $\left(x-x_{0}\right) / r$ with $x,\left(y-x_{0}\right) / r$ with $y$, and $\left(z-x_{0}\right) / r$ with $z$ to discover

$$
\begin{aligned}
A=\frac{r^{2}}{4 \pi^{2}} \int_{B\left(-x_{0} / r, R / r\right)} & \int_{B\left(-x_{0} / r, R / r\right)} w\left(x_{0}+r y\right) w\left(x_{0}+r z\right) \\
& \times\left(\int_{\mathbb{R}^{2}} \frac{x_{1}-y_{1}}{|x-y|^{2}} \frac{x_{2}-z_{2}}{|x-z|^{2}} \eta(x) d x\right) d y d z
\end{aligned}
$$


For fixed $y, z \in \mathbb{R}^{2}$ and $y \neq z$, let

$$
B \equiv \int_{\mathbb{R}^{2}} \frac{x_{1}-y_{1}}{|x-y|^{2}} \frac{x_{2}-z_{2}}{|x-z|^{2}} \eta(x) d x
$$

Then by the estimation in [3, p. 202-204], we have

$$
|B| \leq c(1+|y|)^{-1}(1+|z|)^{-1}
$$

Substituting this to $(2.3)$ and noting that $w \geq 0$, we obtain

$$
\begin{aligned}
|A| & \leq c r^{2} \int_{B\left(-x_{0} / r, R / r\right)} \int_{B\left(-x_{0} / r, R / r\right)} \frac{w\left(x_{0}+r y\right)}{1+|y|} \frac{w\left(x_{0}+r z\right)}{1+|z|} d y d z \\
& =c\left(r \int_{B\left(-x_{0} / r, R / r\right)} \frac{w\left(x_{0}+r y\right)}{1+|y|} d y\right)^{2} .
\end{aligned}
$$

We restore the original variables by replacing $x_{0}+r y$ with $y$ to obtain

$$
|A| \leq c\left(\int_{B(0, R)} \frac{w(y)}{r+\left|y-x_{0}\right|} d y\right)^{2}
$$

Therefore,

$$
\left(v_{x_{1}} v_{x_{2}}\right)^{* *}\left(x_{0}\right)=\sup _{0<r \leq 1}|A| \leq c\left(\int_{B(0, R)} \frac{w(y)}{\left|y-x_{0}\right|} d y\right)^{2}
$$

For $x_{0} \in \mathbb{R}^{2}$, we next write

$$
g\left(x_{0}\right)=\int_{B(0, R)} \frac{w(y)}{\left|y-x_{0}\right|} d y
$$

and claim

$$
g \in \dot{K}_{2 q, \operatorname{loc}}^{1-1 / q, 2}\left(\mathbb{R}^{2}\right) .
$$


To this end, first choose a cutoff function $\rho \in C_{c}^{\infty}\left(\mathbb{R}^{2}\right)$, with $0 \leq \rho \leq 1$, $\rho=1$ on $B(0, R), \rho \equiv 0$ on $\mathbb{R}^{2} \backslash B(0,2 R),|D \rho| \leq c / R$. Choose also $\lambda_{\varepsilon} \in C_{c}^{\infty}\left(\mathbb{R}^{2}\right)$ satisfying $0 \leq \lambda_{\varepsilon} \leq 1, \lambda_{\varepsilon}=0$ on $B\left(x_{0}, \varepsilon\right), \lambda_{\varepsilon}=1$ on $\mathbb{R}^{2} \backslash B\left(x_{0}, 2 \varepsilon\right),\left|D \lambda_{\varepsilon}\right| \leq c / \varepsilon$. Let $M(f)$ denote the Hardy-Littlewood maximal function of $f$. By [3], we have

$$
\begin{aligned}
0 \leq g\left(x_{0}\right) \leq & \int_{\mathbb{R}^{2}} \rho(y) \frac{w(y)}{\left|y-x_{0}\right|} d y \\
\leq & -p \cdot v \cdot \int_{\mathbb{R}^{2}} \rho(y) D u(y) \cdot \frac{y-x_{0}}{\left|y-x_{0}\right|^{3}} d y+c M\left(\left.|D u|\right|_{B(0,2 R)}\right)\left(x_{0}\right) \\
& +c \int_{B(0,2 R)}|D u(y)| \frac{1}{\left|y-x_{0}\right|} d y \\
\equiv & g_{1}\left(x_{0}\right)+g_{2}\left(x_{0}\right)+g_{3}\left(x_{0}\right) .
\end{aligned}
$$

Since $y /|y|^{3}$ is a Calderón-Zygmund kernel and $-1 / q<1-1 / q<2(1-$ $1 /(2 q))$, by Corollary 2.1 in $[\mathbf{1 0}]$, we have

$$
\begin{aligned}
\left\|g_{1}\right\|_{\dot{K}_{2 q}^{1-1 / q, 2}\left(\mathbb{R}^{2}\right)} \leq c\|\rho|D u|\|_{\dot{K}_{2 q}^{1-1 / q, 2}\left(\mathbb{R}^{2}\right)} \\
\leq c\left\|\chi_{B(0,2 R)}|D u|\right\|_{\dot{K}_{2 q}^{1-1 / q, 2}\left(\mathbb{R}^{2}\right)} .
\end{aligned}
$$

Furthermore, by the same corollary in $[\mathbf{1 0}]$, we have

$$
\left\|g_{2}\right\|_{\dot{K}_{2 q}^{1-1 / q, 2}\left(\mathbb{R}^{2}\right)} \leq c\left\|\chi_{B(0,2 R)}|D u|\right\|_{\dot{K}_{2 q}^{1-1 / q, 2}\left(\mathbb{R}^{2}\right)} .
$$

Now, let $k_{0} \in \mathbb{Z}$ such that $2^{k_{0}} \leq R / 2<2^{k_{0}+1}$. Therefore, $2^{k_{0}+2} \leq 2 R<$ $2^{k_{0}+3}$. Write

$$
\begin{aligned}
g_{3}\left(x_{0}\right) & =c \int_{\mathbb{R}^{2}}|D u(y)| \frac{1}{\left|y-x_{0}\right|} \chi_{B(0,2 R)}(y) d y \\
& \leq \sum_{l=-\infty}^{k_{0}+3} \int_{\mathbb{R}^{2}}|D u(y)| \chi_{l}(y) \frac{1}{\left|y-x_{0}\right|} d y \\
& =\sum_{l=-\infty}^{k_{0}+3} \int_{\mathbb{R}^{2}}\left|D u\left(y+x_{0}\right)\right| \chi_{l}\left(y+x_{0}\right) \frac{1}{|y|} d y \\
& \equiv c \sum_{l=-\infty}^{k_{0}+3} \bar{g}_{l}\left(x_{0}\right) .
\end{aligned}
$$


Let $\varepsilon>0$ to be determined late. We have

$\left\|\chi_{B(0, R / 2)} g_{3}\right\|_{\dot{K}_{2 q}^{1-1 / q, 2}\left(\mathbb{R}^{2}\right)}$

$$
\begin{aligned}
& \leq c\left\{\sum_{k=-\infty}^{k_{0}+1} 2^{2 k(1-1 / q)}\left(\sum_{l=-\infty}^{k_{0}+3}\left\|\chi_{k} \bar{g}_{l}\right\|_{L^{2 q}\left(\mathbb{R}^{2}\right)}\right)^{2}\right\}^{1 / 2} \\
& \leq c\left\{\sum_{k=-\infty}^{k_{0}+1} 2^{2 k(1-1 / q)}\left(\sum_{l=-\infty}^{k_{0}+3} 2^{-2 \varepsilon l}\left\|\chi_{k} \bar{g}_{l}\right\|_{L^{2 q}\left(\mathbb{R}^{2}\right)}^{2} 2^{2 \varepsilon k_{0}}\right)\right\}^{1 / 2} \\
& \leq c(R)\left\{\sum_{l=-\infty}^{k_{0}+3} \sum_{k=-\infty}^{\min \left\{l+2, k_{0}+1\right\}} 2^{2 k(1-1 / q)-2 \varepsilon l}\left\|_{k}^{\chi} \bar{g}_{l}\right\|_{L^{2 q}\left(\mathbb{R}^{2}\right)}^{2}\right\}^{1 / 2} \\
& +c(R)\left\{\sum_{l=-\infty}^{k_{0}-2} \sum_{k=l+3}^{k_{0}+1} 2^{2 k(1-1 / q)-2 \varepsilon l}\left\|\chi_{k} \bar{g}_{l}\right\|_{L^{2 q}\left(\mathbb{R}^{2}\right)}^{2}\right\}^{1 / 2} \\
& \equiv J_{1}+J_{2} .
\end{aligned}
$$

For $J_{1}$, we first note that when $x_{0} \in A_{k}$ and $k \leq l+2$, by the definition of $\bar{g}_{l}\left(x_{0}\right)$, we have

$$
\left|\bar{g}_{l}\left(x_{0}\right)\right| \leq \int_{\mathbb{R}^{2}}\left|D u\left(y+x_{0}\right)\right| \chi_{l}\left(y+x_{0}\right) \frac{1}{|y|} \chi_{\left\{y \in \mathbb{R}^{2}:|y| \leq 2^{l+3}\right\}}(y) d y .
$$

Thus,

$$
\begin{aligned}
\left\|\chi_{l} \bar{g}_{l}\right\|_{L^{2 q}\left(\mathbb{R}^{2}\right)} & \leq\left\||D u| \chi_{l}\right\|_{L^{2 q}\left(\mathbb{R}^{2}\right)} \int_{|y| \leq 2^{l+3}} \frac{1}{|y|} d y \\
& \leq c 2^{l}\left\||D u| \chi_{l}\right\|_{L^{2 q}\left(\mathbb{R}^{2}\right)} .
\end{aligned}
$$

By choosing $0<\varepsilon<1$, we obtain

$$
\begin{aligned}
J_{1} & \leq c\left\{\sum_{l=-\infty}^{k_{0}+3} 2^{2(1-\varepsilon) l}\left\||D u| \chi_{l}\right\|_{L^{2 q}\left(\mathbb{R}^{2}\right)}^{2}\left(\sum_{k=-\infty}^{l+2} 2^{2 k(1-1 / q)}\right)\right\}^{1 / 2} \\
& \leq c\left\{\sum_{l=-\infty}^{k_{0}+3} 2^{2 l(1-1 / q)}\left\||D u| \chi_{l}\right\|_{L^{2 q}\left(\mathbb{R}^{2}\right)}^{2}\right\}^{1 / 2} \\
& \leq c\left\|\chi_{B(0,4 R)}|D u|\right\|_{\dot{K}_{2 q}^{1-1 / q, 2}\left(\mathbb{R}^{2}\right)} .
\end{aligned}
$$


On $J_{2}$, we note that when $k \geq l+3$ and $x_{0} \in A_{k}$,

$$
\begin{aligned}
\bar{g}_{l}\left(x_{0}\right) & \leq \int_{\mathbb{R}^{2}}\left|D u\left(x_{0}+y\right)\right| \chi_{l}\left(x_{0}+y\right) \frac{1}{|y|} \chi_{\left\{y: \frac{3}{4}\left|x_{0}\right| \leq|y| \leq \frac{5}{4}\left|x_{0}\right|\right\}}(y) d y \\
& \leq c 2^{-k} \int_{\mathbb{R}^{2}}\left|D u\left(x_{0}+y\right)\right| \chi_{l}\left(x_{0}+y\right) d y \\
& \leq c 2^{-k+2 l(1-1 /(2 q))}\left\||D u| \chi_{l}\right\|_{L^{2 q}\left(\mathbb{R}^{2}\right)} .
\end{aligned}
$$

Therefore,

$$
\begin{aligned}
J_{2} & \leq c\left\{\sum_{l=-\infty}^{k_{0}-2} 2^{2 l(1-1 / q)}\left\||D u| \chi_{l}\right\|_{L^{2 q}\left(\mathbb{R}^{2}\right)}^{2}\left(\sum_{k=l+3}^{k_{0}+1} 2^{2 l(1-\varepsilon)}\right)\right\}^{1 / 2} \\
& \leq c\left\{\sum_{l=-\infty}^{k_{0}-2} 2^{2 l(1-1 / q)}\left\||D u| \chi_{l}\right\|_{L^{2 q}\left(\mathbb{R}^{2}\right)}^{2}\left(\sum_{k=l+3}^{k_{0}+1} 2^{2 k(1-\varepsilon)}\right)\right\}^{1 / 2} \\
& \leq c\left\{\sum_{l=-\infty}^{k_{0}-2} 2^{2 l(1-1 / q)}\left\||D u| \chi_{l}\right\|_{L^{2 q}\left(\mathbb{R}^{2}\right)}^{2}\right\}^{1 / 2} \\
& \leq c\left\|\chi_{B(0,4 R)}|D u|\right\|_{\dot{K}_{2 q}^{1-1 / q, 2}\left(\mathbb{R}^{2}\right)} .
\end{aligned}
$$

Substituting the estimations on $J_{1}$ and $J_{2}$ into (2.8), we have

$$
\left\|\chi_{B(0, R / 2)} g_{3}\right\|_{\dot{K}_{2 q}^{1-1 / q, 2}\left(\mathbb{R}^{2}\right)} \leq c\left\|\chi_{B(0,4 R)}|D u|\right\|_{\dot{K}_{2 q}^{1-1 / q, 2}\left(\mathbb{R}^{2}\right)} .
$$

By combining (2.6), (2.7) and (2.9), we obtain

$$
\left\|\chi_{B(0, R / 2)} g\right\|_{\dot{K}_{2 q}^{1-1 / q, 2}\left(\mathbb{R}^{2}\right)} \leq c\left\|\chi_{B(0,4 R)}|D u|\right\|_{\dot{K}_{2 q}^{1-1 / q, 2}\left(\mathbb{R}^{2}\right)}<\infty .
$$

Thus, the claim (2.5) holds. From (2.10) and (2.4), we deduce

(2.11) $\left\|\chi_{B(0, R / 2)}\left(v_{x_{1}} v_{x_{2}}\right)^{* *}\right\|_{\dot{K}_{2 q}^{2(1-1 / q), 1}\left(\mathbb{R}^{2}\right)}$

$$
\leq c(R)\left\|\chi_{B(0,4 R)}|D u|\right\|_{\dot{K}_{2 q}^{1-1 / q, 2}\left(\mathbb{R}^{2}\right)}^{2} .
$$

Now recall from (2.1) that $\theta=u-v$ is harmonic and thus smooth within $B(0, R)$. Consequently, if $x_{0} \in B(0, R / 2)$, by a similar procedure to $[\mathbf{1 0}$, 
p. 205], we obtain that $\left(\theta_{x_{1}} \theta_{x_{2}}\right)^{* *}\left(x_{0}\right) \leq c \sup _{x \in B(0,3 R / 4)}|D \theta(x)|^{2}$; and

$$
\left.\begin{array}{rl}
\sup _{B(0,3 R / 4)}|D \theta| \leq & c(R) \int_{B(0, R)}|D u(x)| d x \\
& +\frac{1}{|B(0, R)|} \int_{B(0, R)}|v(x)| d x \\
\leq & c(R) \int_{B(0, R)}|D u(x)| d x+c(R) \int_{B(0, R)} w(x) d x \\
\leq & c(R) \int_{B(0,2 R)}|D u(x)| d x \\
\leq & c(R) \sum_{l=-\infty}^{k_{0}+3} \int_{\mathbb{R}^{2}}|D u(x)| \chi_{l}(x) d x \\
\leq & c(R) \sum_{l=-\infty}^{k_{0}+3} 2^{2 l(1-1 /(2 q))}\left\||D u| \chi_{l}\right\|_{L^{2 q}\left(\mathbb{R}^{2}\right)} \\
\leq & c(R) 2^{k_{0}}\left\{\sum_{l=-\infty}^{k_{0}+3} 2^{2 l(1-1 / q)}\left\||D u|_{l}\right\|_{L^{2 q}\left(\mathbb{R}^{2}\right)}^{2}\right.
\end{array}\right\}^{1 / 2}
$$

Thus,

$$
\begin{aligned}
\| \chi_{B(0, R / 2)} & \left(\theta_{x_{1}} \theta_{x_{2}}\right)^{* *} \|_{\dot{K}_{q}^{2(1-1 / q), 1}\left(\mathbb{R}^{2}\right)} \\
& \leq c\left(\sum_{l=-\infty}^{k_{0}+1} 2^{2 l}\right)\left\|\chi_{B(0,4 R)}|D u|\right\|_{\dot{K}_{2 q}^{1-1 / q, 2}\left(\mathbb{R}^{2}\right)}^{2} \\
& \leq c\left\|\chi_{B(0,4 R)}|D u|\right\|_{\dot{K}_{2 q}^{1-1 / q, 2}\left(\mathbb{R}^{2}\right)}^{2}
\end{aligned}
$$

Similarly, if $x_{0} \in B(0, R / 2)$, by (2.12), we have

$$
\begin{aligned}
\left(\theta_{x_{1}} v_{x_{2}}\right)^{* *}\left(x_{0}\right)= & \sup _{0<r \leq 1}\left|\frac{1}{r^{2}} \int_{\mathbb{R}^{2}} \theta_{x_{1}} v_{x_{2}} \eta\left(\frac{x-x_{0}}{r}\right) d x\right| \\
\leq & c \sup _{B(0,3 R / 4)}|D \theta|^{2}+c\left(\sup _{B(0,3 R / 4)}|D \theta|\right) M\left(\chi_{B(0, R)}|D u|\right)\left(x_{0}\right) \\
\leq & c\left\|\chi_{B(0,4 R)}|D u|\right\|_{\dot{K}_{2 q}^{1-1 / q, 2}\left(\mathbb{R}^{2}\right)}^{2} \\
& +c\left\|\chi_{B(0,4 R)}|D u|\right\|_{\dot{K}_{2 q}^{1-1 / q, 2}\left(\mathbb{R}^{2}\right)} M\left(\chi_{B(0, R)}|D u|\right)\left(x_{0}\right) .
\end{aligned}
$$


Now, Corollary 2.1 in $[\mathbf{1 0}]$ gives us that

$$
\begin{aligned}
\| \chi_{B(0, R / 2)}( & \left.\theta_{x_{1}} v_{x_{2}}\right)^{* *} \|_{\dot{K}_{q}^{2(1-1 / q), 1}\left(\mathbb{R}^{2}\right)} \\
\leq & c\left\|\chi_{B(0,4 R)}|D u|\right\|_{\dot{K}_{2 q}^{1-1 / q, 2}\left(\mathbb{R}^{2}\right)}^{2}+c\left\|\chi_{B(0,4 R)}|D u|\right\|_{\dot{K}_{2 q}^{1-1 / q, 2}\left(\mathbb{R}^{2}\right)} \\
& \times\left(\sum_{k=-\infty}^{k_{0}+1} 2^{2 k(1-1 / q)}\left\|\chi_{k} M\left(\chi_{B(0, R)}|D u|\right)\right\|_{L^{q}\left(\mathbb{R}^{2}\right)}\right) \\
\leq & c\left\|\chi_{B(0,4 R)}|D u|\right\|_{\dot{K}_{2 q}^{1-1 / q, 2}\left(\mathbb{R}^{2}\right)}^{2}+c\left\|\chi_{B(0,4 R)}|D u|\right\|_{\dot{K}_{2 q}^{1-1 / q, 2}\left(\mathbb{R}^{2}\right)} \\
& \times\left(\sum_{k=-\infty}^{k_{0}+1} 2^{k(1-1 / q)+k}\left\|\chi_{k} M\left(\chi_{B(0, R)}|D u|\right)\right\|_{L^{2 q}\left(\mathbb{R}^{2}\right)}\right) \\
\leq & c\left\|\chi_{B(0,4 R)}|D u|\right\|_{\dot{K}_{2 q}^{1-1 / q, 2}\left(\mathbb{R}^{2}\right)}^{2} \\
& +c\left\|\chi_{B(0,4 R)}|D u|\right\|_{\dot{K}_{2 q}^{1-1 / q, 2}\left(\mathbb{R}^{2}\right)}\left\|M\left(\chi_{B(0, R)}|D u|\right)\right\|_{\dot{K}_{2 q}^{1-1 / q, 2}\left(\mathbb{R}^{2}\right)} \\
\leq & c\left\|\chi_{B(0,4 R)}|D u|\right\|_{\dot{K}_{2 q}^{1-1 / q, 2}\left(\mathbb{R}^{2}\right)}^{2} .
\end{aligned}
$$

The same argument shows that

(2.15) $\left\|\chi_{B(0, R / 2)}\left(v_{x_{1}} \theta_{x_{2}}\right)^{* *}\right\|_{\dot{K}_{q}^{2(1-1 / q), 1}\left(\mathbb{R}^{2}\right)}$

$$
\leq c\left\|\chi_{B(0,4 R)}|D u|\right\|_{\dot{K}_{2 q}^{1-1 / q, 2}\left(\mathbb{R}^{2}\right)}^{2} .
$$

By combining the estimations (2.11), (2.13)-(2.15), we obtain

$\left\|\chi_{B(0, R / 2)}\left(u_{x_{1}} u_{x_{2}}\right)^{* *}\right\|_{\dot{K}_{q}^{2(1-1 / q), 1}\left(\mathbb{R}^{2}\right)} \leq c\left\|\chi_{B(0,4 R)}|D u|\right\|_{\dot{K}_{2 q}^{1-1 / q, 2}\left(\mathbb{R}^{2}\right)}^{2}<\infty$

for each $R \geq 8$. Hence $u_{x_{1}} u_{x_{2}} \in \mathcal{H}_{\mathrm{loc}} \dot{K}_{q}^{2(1-1 / q), 1}\left(\mathbb{R}^{2}\right)$.

By rotating variables, similar to $[\mathbf{3}]$, we can prove that $u_{x_{1}}^{2}-u_{x_{2}}^{2} \in$ $\mathcal{H}_{\text {loc }} \dot{K}_{q}^{2(1-1 / q), 1}\left(\mathbb{R}^{2}\right)$. Finally, estimate (1.2) is a consequence of Proposition 1.

This finishes the proof of Theorem 1. 
Proof of Theorem 2: We have the ordinary differential equation

$$
-\frac{1}{r}\left(r u^{\prime}\right)^{\prime}(r)=w(r)
$$

We only prove the estimates for $u_{x_{1}} u_{x_{2}}$, because, similar to [3], those for $u_{x_{1}}^{2}-u_{x_{2}}^{2}$ follow then by performing a rotation. Let $\eta \in C_{c}^{\infty}(B(0,1))$, $\eta \geq 0$ and $\int_{B(0,1)} \eta(x) d x=1$. Define

$$
f(x)=\left(u_{x_{1}} u_{x_{2}}\right)(x)=\frac{x_{1} x_{2}}{r^{2}}\left(u^{\prime}\right)^{2}(r) \quad \text { and } \quad f_{i}=f \chi_{i} \quad \text { for } \quad i \in \mathbb{Z}
$$

We also write

$$
f^{* *}(x)=\sup _{0<r \leq 1}\left|\frac{1}{r^{2}} \int_{\mathbb{R}^{2}} \eta\left(\frac{x-y}{r}\right) f(y) d y\right|
$$

and for $i \in \mathbb{Z}$,

$$
f_{i}^{* *}(x)=\sup _{0<r \leq 1}\left|\frac{1}{r^{2}} \int_{\mathbb{R}^{2}} \eta\left(\frac{x-y}{r}\right) f_{i}(y) d y\right| .
$$

Since $w \in \dot{K}_{q_{2}, \text { loc }}^{2\left(1-1 / q_{2}\right), 1}\left(\mathbb{R}^{2}\right) \subset L_{\text {loc }}^{1}\left(\mathbb{R}^{2}\right)$, the ordinary differential equation (2.16) implies that $v(r) \equiv r u^{\prime}(r)$ is absolutely continuous on each interval $[0, R]$. In particular, if $2^{i-1} \leq r \leq 2^{i}$,

$$
\left|v(r)-\frac{1}{2^{i-1}} \int_{2^{i-1}}^{2^{i}} v(r) d r\right| \leq \int_{2^{i-1}}^{2^{i}}\left|v^{\prime}(r)\right| d r \leq \int_{2^{i-1}}^{2^{i}} r|w(r)| d r
$$

It is easy to verify that if $h \in L^{q}\left(\mathbb{R}^{2}\right), \operatorname{supp} h \subseteq B(0, r)$ and $\int_{\mathbb{R}^{2}} h(x) d x=0$, then $|B(0, r)|^{1 / q-1}\|h\|_{L^{q}\left(\mathbb{R}^{2}\right)}^{-1} h$ is a central $(2(1-1 / q), q)$-atom supported in $B(0, r)$; see [11]. Thus, by Theorem 2.1 in [12] (see also [6]),

$$
\begin{aligned}
\left\|h^{* *}\right\|_{\dot{K}_{q}^{2(1-1 / q), 1}\left(\mathbb{R}^{2}\right)} & \leq\left\|h^{*}\right\|_{\dot{K}_{q}^{2(1-1 / q), 1}\left(\mathbb{R}^{2}\right)} \\
& \leq c r^{2(1-1 / q)}\|h\|_{L^{q}\left(\mathbb{R}^{2}\right)} \\
& \leq c r^{2}\|h\|_{L^{\infty}\left(\mathbb{R}^{2}\right)},
\end{aligned}
$$

if $h \in L^{\infty}\left(\mathbb{R}^{2}\right)$. Therefore,

$$
\left\|f_{i}^{* *}\right\|_{\dot{K}_{q}^{2(1-1 / q), 1}\left(\mathbb{R}^{2}\right)} \leq c 2^{2 i}\left\|f_{i}\right\|_{L^{\infty}\left(\mathbb{R}^{2}\right)} .
$$


Moreover, $f_{i+2}^{* *}(x)=0$ on $B\left(0,2^{i+1}-1\right) \supset B\left(0,2^{i}\right)$ if $i \geq 1$. Now, by (2.17),

$$
\begin{aligned}
2^{2 i}\left\|f_{i}\right\|_{L^{\infty}\left(\mathbb{R}^{2}\right)} & \leq 4 \sup _{2^{i-1}<r \leq 2^{i}} r^{2}\left|u^{\prime}(r)\right|^{2} \\
& \leq c\left\{2^{-i} \int_{2^{i-1}}^{2^{i}}\left|r u^{\prime}(r)\right| d r+\int_{2^{i-1}}^{2^{i}}\left|\left(r u^{\prime}(r)\right)^{\prime}\right| d r\right\}^{2} \\
& \leq c\left\{2^{-i / q_{1}} \int_{2^{i-1}}^{2^{i}}\left|r^{1 / q_{1}} u^{\prime}(r)\right| d r+\int_{2^{i-1}}^{2^{i}} r|w(r)| d r\right\}^{2} \\
& \leq c\left\{2^{i\left(1-2 / q_{1}\right)}\left(\int_{2^{i-1}}^{2^{i}} r\left|u^{\prime}(r)\right|^{q_{1}} d r\right)^{1 / q_{1}}+\int_{A_{i}}|w(x)| d x\right\}^{2} \\
& \leq c\left\{2^{i\left(1-2 / q_{1}\right)}\left(\int_{A_{i}}|D u(x)|^{q_{1}} d x\right)^{1 / q_{1}}+\int_{A_{i}}|w(x)| d x\right\}^{2} .
\end{aligned}
$$

Therefore,

$$
\begin{aligned}
& \left\|\chi_{B_{k}} f^{* *}\right\|_{\dot{K}_{q}^{2(1-1 / q), 1}\left(\mathbb{R}^{2}\right)} \\
& \quad \leq \sum_{j=-\infty}^{\infty}\left\|\chi_{B_{k}} f_{j}^{* *}\right\|_{\dot{K}_{q}^{2(1-1 / q), 1}\left(\mathbb{R}^{2}\right)} \\
& \quad=\sum_{j=-\infty}^{k+2}\left\|\chi_{B_{k}} f_{j}^{* *}\right\|_{\dot{K}_{q}^{2(1-1 / q), 1}\left(\mathbb{R}^{2}\right)} \\
& \quad \leq c \sum_{j=-\infty}^{k+2}\left\{\left(2^{j\left(1-2 / q_{1}\right)}\left\||D u| \chi_{j}\right\|_{\left.\left.L^{q_{1}\left(\mathbb{R}^{2}\right)}\right)^{2}+\left\||w| \chi_{j}\right\|_{L^{1}\left(\mathbb{R}^{2}\right)}^{2}\right\}}\right.\right. \\
& \quad \leq c \sum_{j=-\infty}^{k+2} 2^{4 j\left(1 / 2-1 / q_{1}\right)}\left\||D u| \chi_{j}\right\|_{L^{q_{1}}\left(\mathbb{R}^{2}\right)}^{2}+c \sum_{j=-\infty}^{k+2}\left\||w| \chi_{j}\right\|_{L^{1}\left(\mathbb{R}^{2}\right)}^{2} \\
& \leq c\left\|\chi_{B_{k+2}}|D u|\right\|_{\dot{K}_{q_{1}}^{2\left(1 / 2-1 / q_{1}\right), 2}\left(\mathbb{R}^{2}\right)}^{2}+c\left\|\chi_{B_{k+2}} w\right\|_{\dot{K}_{q_{2}}^{2\left(1-1 / q_{2}\right), 1}\left(\mathbb{R}^{2}\right)}^{2} \\
& \quad<\infty .
\end{aligned}
$$

Hence $u_{x_{1}} u_{x_{2}} \in \mathcal{H}_{\text {loc }} \dot{K}_{q}^{2(1-1 / q), 1}\left(\mathbb{R}^{2}\right)$. The estimation (1.4) now follows from Proposition 1.

This finishes the proof of Theorem 2. 
Proof of Proposition 2: Since $h \dot{K}_{q}^{n(1-1 / q), 1}\left(\mathbb{R}^{n}\right) \subset h^{1}\left(\mathbb{R}^{n}\right)$, (1.4) holds by Theorem 3.1 in $[\mathbf{3}]$. The remaining thing is to verify that $f \in h \dot{K}_{q}^{n(1-1 / q), 1}\left(\mathbb{R}^{n}\right)$. Choose $\phi \in C_{c}^{\infty}\left(\mathbb{R}^{n}\right)$, supp $\phi \subset B(0,1)$ and $\int_{\mathbb{R}^{n}} \phi(x) d x=1$. For $t>0$, set $\phi_{t}(x)=\frac{1}{t^{n}} \phi\left(\frac{x}{t}\right)$. Then by (1.4), for every $x \in \mathbb{R}^{n}$ and $0<t \leq 1$, we have

$$
\phi_{t} * f_{k}(x) \longrightarrow \phi_{t} * f(x), \quad \text { as } \quad k \longrightarrow \infty ;
$$

therefore,

$$
\begin{aligned}
\varliminf_{k \rightarrow \infty} \sup _{1 \geq t>0}\left|\phi_{t} * f_{k}(x)\right| & =\sup _{k} \inf _{l \geq k} \sup _{1 \geq t>0}\left|\phi_{t} * f_{l}(x)\right| \\
& \geq \sup _{1 \geq t>0} \sup _{k} \inf _{l \geq k}\left|\phi_{t} * f_{l}(x)\right| \\
& =\sup _{1 \geq t>0} \varliminf_{k \rightarrow \infty}\left|\phi_{t} * f_{k}(x)\right| \\
& =\sup _{1 \geq t>0}\left|\phi_{t} * f(x)\right| .
\end{aligned}
$$

On the other hand, since $\left\|f_{k}\right\|_{h \dot{K}_{q}^{n(1-1 / q), 1}\left(\mathbb{R}^{n}\right)} \leq c$, it follows from Theorem 2.1 in $[4]$ that

$$
\left\|\sup _{1 \geq t>0}\left|\phi_{t} * f_{k}\right|\right\|_{\dot{K}_{q}^{n(1-1 / q), 1}\left(\mathbb{R}^{n}\right)} \leq c .
$$

By Fatou's lemmas of series and integration, we have

$$
\begin{aligned}
& c \geq \lim _{k \rightarrow \infty}\left\|\sup _{1 \geq t>0}\left|\phi_{t} * f_{k}\right|\right\|_{\dot{K}_{q}^{n(1-1 / q), 1}\left(\mathbb{R}^{n}\right)} \\
& =\lim _{k \rightarrow \infty}\left\{\sum_{l=-\infty}^{\infty} 2^{\ln (1-1 / q)}\left\|\chi_{l} \sup _{1 \geq t>0}\left|\phi_{t} * f_{k}\right|\right\|_{L^{q}\left(\mathbb{R}^{n}\right)}\right\} \\
& \geq \sum_{l=-\infty}^{\infty} 2^{\ln (1-1 / q)} \varliminf_{k \rightarrow \infty}\left\|\chi_{l} \sup _{1 \geq t>0}\left|\phi_{t} * f_{k}\right|\right\|_{L^{q}\left(\mathbb{R}^{n}\right)} \\
& \geq \sum_{l=-\infty}^{\infty} 2^{l n(1-1 / q)}\left\|\chi_{l} \varliminf_{k \rightarrow \infty} \sup _{1 \geq t>0}\left|\phi_{t} * f_{k}\right|\right\|_{L^{q}\left(\mathbb{R}^{n}\right)} \\
& \geq \sum_{l=-\infty}^{\infty} 2^{l n(1-1 / q)}\left\|\chi_{l} \sup _{1 \geq t>0}\left|\phi_{t} * f\right|\right\|_{L^{q}\left(\mathbb{R}^{n}\right)} \\
& =\left\|\sup _{1 \geq t>0}\left|\phi_{t} * f\right|\right\|_{\dot{K}_{q}^{n(1-1 / q), 1}\left(\mathbb{R}^{n}\right)} .
\end{aligned}
$$


Again, from Theorem 2.1 in [4], we deduce that $f \in h \dot{K}_{q}^{n(1-1 / q), 1}\left(\mathbb{R}^{n}\right)$.

This finishes the proof of Proposition 2.

\section{References}

1. R. Coifman, P. L. Lions, Y. Meyer and S. Semmes, Compensated compactness and Hardy spaces, J. Math. Pures Appl. 72 (1993), 247-286.

2. J. M. Delort, Existence de nappes de tourbillon en dimension deux, J. Amer. Math. Soc. 4 (1991), 553-586.

3. L. C. Evans And S. Müller, Hardy spaces and the two-dimensional Euler equations with nonnegative vorticity, J. Amer. Math. Soc. 7 (1994), 199-219.

4. D. FAN AND D. YANG, The weighted Herz-type Hardy spaces $h \dot{K}_{q}^{\alpha, p}\left(w_{1}, w_{2}\right)$, Approx. Theory Appl. 13(4) (1997), 19-41.

5. C. Fefferman and E. M. Stein, $\mathcal{H}^{p}$ spaces of several variables, Acta Math. 129 (1972), 137-193.

6. J. García-Cuerva And M.-J. L. Herrero, A theory of Hardy spaces associated to Herz spaces, Proc. London Math. Soc. (3) 69 (1994), 605-628.

7. D. GoldBerg, A local version of real Hardy spaces, Duke Math. J. 46 (1979), 27-42.

8. C. Herz, Lipschitz spaces and Bernstein's theorem on absolutely convergent Fourier transforms, J. Math. Mech. 18 (1968), 283-324.

9. P. W. Jones And J.-L. Journé, On weak convergence in $\mathcal{H}^{1}\left(\mathbb{R}^{d}\right)$, Proc. Amer. Math. Soc. 120 (1994), 137-138.

10. X. Li AND D. YANG, Boundedness of some sublinear operators on Herz spaces, Illinois J. Math. 40 (1996), 484-501.

11. S. LU AND D. YANG, The weighted Herz-type Hardy spaces and its applications, Sci. China Ser. A 38 (1995), 662-673.

12. S. LU AND D. YANG, Some characterizations of weighted Herz-type Hardy spaces and its applications, Acta Math. Sinica (N.S.) 13(1) (1997), 45-58.

13. S. LU AND D. YANG, Oscillatory singular integrals on Hardy spaces associated with Herz spaces, Proc. Amer. Math. Soc. 123 (1995), 1695-1701.

14. S. Lu AND D. YANG, Herz-type Sobolev and Bessel potential spaces and their applications, Sci. China Ser. A 40 (1997), 113-129. 
15. S. MÜLLER, Hardy space methods for nonlinear partial differential equations, Tatra Mt. Math. Publ. 4 (1994), 159-168.

16. S. Semmes, A primer on Hardy spaces, and some remarks on a theorem of Evans and Müller, Comm. Partial Differential Equations 19 (1994), 277-319.

Dashan Fan:

Anhui University and

University of Wisconsin-Milwaukee

Current Address:

Department of Mathematical Sciences

University of Wisconsin-Milwaukee

P.O. Box 413

Milwaukee WI 53201

U.S.A.

e-mail: fan@alpha1.csd.uwm.edu

\author{
Shanzhen Lu and Dachun Yang: \\ Department of Mathematics \\ Beijing Normal University \\ Beijing 100875 \\ THE PEOPLE'S REPUBLIC \\ OF CHINA \\ e-mail: lusz@bnu.edu.cn \\ e-mail: dcyang@bnu.edu.cn
}

Primera versió rebuda el 26 de febrer de 1997,

darrera versió rebuda el 17 de març de 1998 